\title{
Spermatogonial deubiquitinase USP9X is essential for proper spermatogenesis in mice
}

\author{
Kasane Kishi ${ }^{1}$, Aya Uchida ${ }^{1}$, Hinako M Takase ${ }^{2}$, Hitomi Suzuki², Masamichi Kurohmaru ${ }^{1}$, \\ Naoki Tsunekawa1', Masami Kanai-Azuma², Stephen A Wood ${ }^{3}$ and Yoshiakira Kanai ${ }^{1}$ \\ ${ }^{1}$ Department of Veterinary Anatomy, The University of Tokyo, Bunkyo-ku, Tokyo, Japan, ${ }^{2}$ Department of \\ Experimental Animal Model for Human Disease, Centre for Experimental Animals, Tokyo Medical and Dental \\ University, Bunkyo-ku, Tokyo, Japan, and ${ }^{3}$ Griffith Institute for Drug Discovery, Griffith University, Brisbane, \\ Queensland, Australia \\ Correspondence should be addressed to Y Kanai; Email: aykanai@mail.ecc.u-tokyo.ac.jp
}

\begin{abstract}
USP9X (ubiquitin-specific peptidase 9, $\mathrm{X}$ chromosome) is the mammalian orthologue of Drosophila deubiquitinase fat facets that was previously shown to regulate the maintenance of the germ cell lineage partially through stabilizing Vasa, one of the widely conserved factors crucial for gametogenesis. Here, we demonstrate that USP9X is expressed in the gonocytes and spermatogonia in mouse testes from newborn to adult stages. By using Vasa-Cre mice, germ cell-specific conditional deletion of Usp9x from the embryonic stage showed no abnormality in the developing testes by 1 week and no appreciable defects in the undifferentiated and differentiating spermatogonia at postnatal and adult stages. Interestingly, after 2 weeks, Usp9x-null spermatogenic cells underwent apoptotic cell death at the early spermatocyte stage, and then, caused subsequent aberrant spermiogenesis, which resulted in a complete infertility of Usp9x conditional knockout male mice. These data provide the first evidence of the crucial role of the spermatogonial USP9X during transition from the mitotic to meiotic phases and/or maintenance of early meiotic phase in Usp9x conditional knockout testes. Reproduction (2017) 154 135-143
\end{abstract}

\section{Introduction}

In mammalian testes, spermatozoa are constantly produced by well-balanced process between the maintenance of undifferentiated spermatogonia including spermatogenic stem/progenitor cells and their differentiation into differentiating spermatogonia in the basal compartment of the seminiferous epithelia. Subsequently, the differentiating spermatogonia undergo meiotic initiation, leading to the cyclical differentiation into spermatocytes, round and elongated spermatids and spermatozoa in the adluminal compartment of the seminiferous tubules (Russell et al. 1990). During the postnatal development of mouse testes, a certain population of the gonocytes, the precursors of spermatogonia, directly undergoes the 1st wave of spermatogenesis, followed by the initial onset of meiosis by 2 weeks at the adluminal compartment. The remaining population of the gonocytes is differentiated into the spermatogenic stem/progenitor cells within the basal compartment, which subsequently contribute to the 2nd and later cycles of the spermatogenesis throughout the reproductive life ( 1.5-year-old mice) (Yoshida et al. 2006).

The ubiquitin-protease degradation system is well known to be involved in various steps of spermatogenesis including the maintenance/proliferation of the spermatogonia, meiotic entry, chromosomal recombination, acrosome biogenesis and spermiogenesis (Hou \& Yang 2013). For example, the deletion of Ubb, the polyubiquitin gene with predominant expression in male germ cells, resulted in the complete failure of meiotic progression of spermatocytes beyond early pachytene stage as well as more than $50 \%$ reduction of ubiquitin concentration in mouse testes (Ryu et al. 2008). It is well known that ubiquitin proteasome-related genes including ubiquitin-ligase and deubiquitinase are stage-specifically expressed in the spermatogenic cells and they appear to be involved in multiple steps of spermatogenesis (review by Bose et al. 2014). The murine deubiquitinase genes such as Usp2, Usp14, Uch/1 and Cyld in male germ cells were also shown to be crucial for the proper spermatogenesis by a number of forward genetic approaches of deletion mutants (Suresh et al. 2015). These findings suggest that the quantity of germ cell components may be post-transcriptionally regulated by the balance of the ubiquitin-ligase and deubiquitinase activities in vivo.

It has been reported that the Drosophila deubiquitinating enzyme fat facets (Faf) is expressed in the germ cell progenitors (pole cells) in fly embryos 
(Fischer-Vize et al. 1992). Later, it was revealed that Faf interacts with a DEAD-box RNA helicase Vasa, one of the widely conserved factors for gametogenesis (Lasko 2013), and protects Vasa from degradation. Indeed, a loss of Faf function displayed defective oogenesis (Liu et al. 2003). The mammalian homologues of Faf are two ubiquitin-specific peptidase genes located on sex chromosomes, Usp9x (ubiquitin-specific peptidase 9, X chromosome, also known as Fam) and Usp9y (ubiquitin-specific peptidase $9, \mathrm{Y}$ chromosome). The USP9Y gene was previously shown to be dispensable for spermatogenesis and male fertility in humans (Luddi et al. 2009). The murine Usp9y gene also appears to be not essential, at least for haploid germ cell formation, because $\mathrm{XO}^{\text {Sry/Eif2s3y }}$ male mice with a complete lack of Usp9y produced offspring in assisted reproduction (Yamauchi et al. 2014). In contrast, murine Usp9x is considered to be a functional orthologue of faf, since it is able to functionally replace faf in Drosophila embryos (Chen et al. 2000). USP9X expression was previously shown in mouse primordial germ cells (PGCs) after 10.5 days post coitum (dpc) (Noma et al. 2002). However, the in vivo function of USP9X in mammalian gametogenesis remains unclear.

In this study, in order to clarify the in vivo role of USP9X in mouse testicular germ cells, we examined the expression patterns of USP9X in mouse spermatogenesis and the phenotype of the Usp9x-null spermatogenic cells by using a germ cell-specific conditional knockout (cKO) approach with Vasa-Cre mice.

\section{Materials and methods}

\section{Animals}

C57BL/6 mice (Japan SLC Inc., Hamamatsu, Japan) were used for immunohistochemical analysis. Usp $9 x^{\text {loxP/loxP }}$ female mice (Stegeman et al. 2013) were crossed with Vasa-Cre male mice (Gallardo et al. 2007). Vasa-Cre males induce recombination in germ cells between $15 \mathrm{dpc}$ and $18 \mathrm{dpc}$ (Cre efficiency was confirmed by crossing with Cre reporter mice (Supplementary data and Supplementary Fig. 1, see section on supplementary data given at the end of this article)), and deletes Usp9x. As Usp9x is located on the $X$ chromosome, the male offspring from this cross that inherited the Vasa-Cre allele lacked Usp9x (Usp9x cKO). Cre-negative males were used as controls (Cnt). Mice were collected at newborn, 1-week-old, 2-week-old, 4-week-old and young and aged adult stages (from 7-weekold to 1-year-old ages). As for the male fertility, the Usp9x cKO and Cnt males (5 week old; 6 males in each genotype) were housed with 2-3 wild-type C57BL/6 females for 7 weeks, and the numbers of wild-type females impregnated by males were calculated.

The animal experiments were carried out in accordance with the Guidelines for Animal Use and Experimentation of the University of Tokyo (approval IDs: P13-764, P14-876, PM15-085, and PH16-084) and the Tokyo Medical and Dental
University (approval IDs: 0140007A, 0150259C2, 0160024C2 and 0170248C2).

\section{Histology and immunohistochemistry}

For histopathological analysis, testes and epididymides were fixed in Bouin's solution or 4\% paraformaldehyde (PFA) and then routinely embedded in paraffin. Sections ( $5 \mu \mathrm{m}$ thickness) were subjected to hematoxylin-eosin (HE) or periodic acidSchiff (PAS) staining. For immunohistochemical staining, testes were fixed in $4 \%$ PFA and routinely embedded in paraffin or OCT compound. The deparaffinized sections $(5 \mu \mathrm{m}$ thickness) were incubated for $12 \mathrm{~h}$ at $4^{\circ} \mathrm{C}$ with anti-Hsc70t (1:2000 dilution; Tsunekawa et al. 1999), anti-Ki-67 (1:100 dilution; Leica Microsystems $\mathrm{GmbH}$ ), anti-promyelocytic leukemia zinc finger (PLZF; formally known as ZBTB16; 1:100 dilution; Calbiochem) and anti-synaptonemal complex protein 3 (SCP3; 1:100 dilution; Santa Cruz Biotechnology) antibodies. The cryosections $(10 \mu \mathrm{m}$ thickness) were also incubated with anti-GDNF family receptor alpha-1 (GFRA1; 1:100 dilution; R\&D Systems), anti-kit oncogene (KIT; 1:100 dilution; R\&D Systems), anti-phospho-histone H2AX (g-H2AX; 1:1000 dilution; Upstate), anti-SCP3 (1:100 dilution; Santa Cruz Biotechnology), anti-SOX9 (1:1,000 dilution; Millipore) and anti-USP9X (1:200 dilution; Kanai-Azuma et al. 2000) antibodies. The immunoreactive signals were visualized by using biotin-conjugated secondary antibodies in combination with the Elite ABC Kit (Vector Laboratories, CA, USA) or using Alexa-488/594-conjugated secondary antibodies (Molecular Probes) in combination with the nuclear counterstaining of 4',6-diamidino-2-phenylindore (DAPI). For negative controls, the sections were incubated with normal IgG (Wako Pure Chemical Industries) instead of the primary antibody, in which any non-specific signals were not detected inside the seminiferous tubules (Supplementary Fig. 2). The paraffin sections were also used for TUNEL assay by using an Apoptotic Detection Kit (Takara). All of the stained samples were analyzed using an Olympus fluorescence microscope (BX51N-34-FL-2; Tokyo, Japan) and Leica TCS SP8 (Leica Microsystems $\mathrm{GmbH}$ ) confocal laser microscope as described previously (Aiyama et al. 2015).

\section{Morphometry}

To assess the incidence of the abnormal seminiferous tubules, only circular seminiferous tubules in transverse sections were used and calculated. SCP3-positive, TUNEL-positive and PLZFand Ki-67-positive cell numbers were counted in 50 circular tubules in each paraffin transverse sections. KIT-positive and SOX9-positive cells were counted in cryosections and the number of KIT-positive cells was divided by the number of SOX9-positive cells in the same section.

\section{Whole-mount immunohistochemistry}

Whole-mount immunohistochemistry was performed as described previously (Aiyama et al. 2015). In brief, the PFAfixed seminiferous tubules were incubated for $12 \mathrm{~h}$ at $4^{\circ} \mathrm{C}$ 
with anti-GFRA1 antibody (1:100 dilution; R\&D Systems). The signals were visualized with Alexa-594-conjugated secondary antibody. All the stained samples were analyzed using an Olympus fluorescence microscope (BX51N-34-FL-2).

\section{Statistical analyses}

Quantitative data are represented as the mean \pm standard error (S.E.). Data were analyzed by Student's $t$-test.

\section{Results}

\section{USP9X expression in the spermatogonia of postnatal and adult testes}

The present immunostaining using anti-USP9X antibody allowed us to detect considerable signal in the cytoplasm of the spermatogenic cells from newborn to adult stages (Fig. 1). The signal quality and antibody specificity were confirmed by a complete loss of the signals in the spermatogenic cells of Usp9x conditional knockout (cKO) testes (Vasa-Cre; Usp9x $x^{\text {loxp/Y }}$ ) (Fig. 1B and G).

In the newborn testes, USP9X-positive gonocytes were surrounded by Sertoli cells (Fig. $1 \mathrm{~A}$ and $\mathrm{A}^{\prime}$ ), and they were then relocated to the basal compartment of the seminiferous tubules by 1 week old (Fig. 1C). At 2 week old, USP9X-positive signals were weakly detectable in the early spermatocytes located in the central region (red arrows in Fig. 1D) that become positive for anti-SCP3 staining (a synaptonemal complex marker specific for the spermatocytes (Yuan et al. 2000)), as well as in the spermatogonia in the basal compartment (white arrows in Fig. 1D). At 4-week-old and adult stages, USP9Xpositive signals were restricted to the spermatogonial population located within the basal compartment (Fig. 1E and F), but not in the pachytene spermatocytes, round spermatids and elongated spermatids (Fig. $1 \mathrm{~F}^{\prime}$ ). Double staining of USP9X and GFRA1 (a specific marker for undifferentiated spermatogonia including spermatogenic stem/progenitor cells (Hara et al. 2014)) or KIT (a marker for differentiating spermatogonia (Zhang et al. 2011)) revealed that USP9X-positive signals were found in both GFRA1-positive and KITpositive populations of the spermatogonia in the adult testes (Fig. $1 \mathrm{H}$ and I). These data indicate that USP9X is expressed predominantly in the spermatogonia and weakly in early spermatocytes, but not in the pachytene spermatocytes and later stage spermatogenic cells.

\section{Small-sized testes with almost complete lack of epididymal spermatozoa, leading to the complete infertility in Usp9x germ cell-specific conditional knockout males}

In order to clarify the in vivo role of Usp9x in spermatogenic cells, the Vasa-Cre; Usp9x ${ }^{\text {loxply }}$ (cKO)

\section{USP9X DAPI}
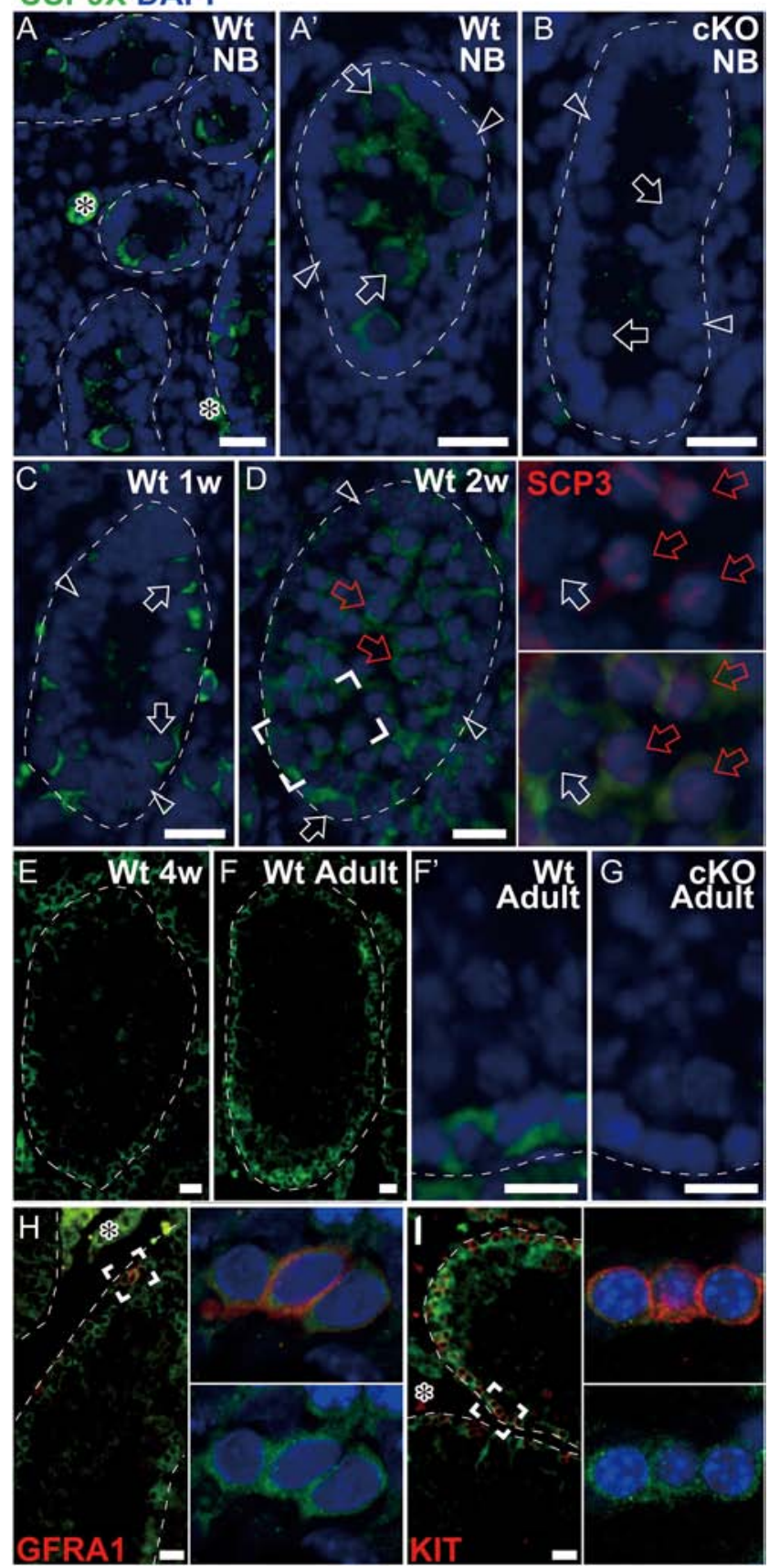

Figure 1 Expression of USP9X during spermatogenesis. Anti-USP9X (green) and anti-SCP3 (D)/GFRA1 (H)/KIT (I) (red) double staining of cryosections in each stage (A, B: newborn, C: 1-week, D: 2-week, E: 4-week, F, G, H and I: adult). DNA was stained by DAPI. A, C, D, $\mathrm{E}, \mathrm{F}, \mathrm{H}$ and $\mathrm{I}$ are sections of wild-type mice $(\mathrm{Wt}) . \mathrm{A}^{\prime}$ and $\mathrm{F}^{\prime}$ are the higher magnified images in newborn and adult stages respectively. $B$ and $G$ are sections of Usp9x conditional knockout mouse (cKO). In the inset of $\mathrm{D}$, white and red arrows indicate SCP3-negative and positive spermatogenic cells respectively. In D, H and I, insets are the higher magnified images indicated by broken rectangles. Broken line, outline of seminiferous tubule; ${ }^{*}$, signals outside the seminiferous tubules; arrow, nucleus of germ cell; arrowhead, nucleus of Sertoli cell. Scale bars, $20 \mu \mathrm{m}$. 

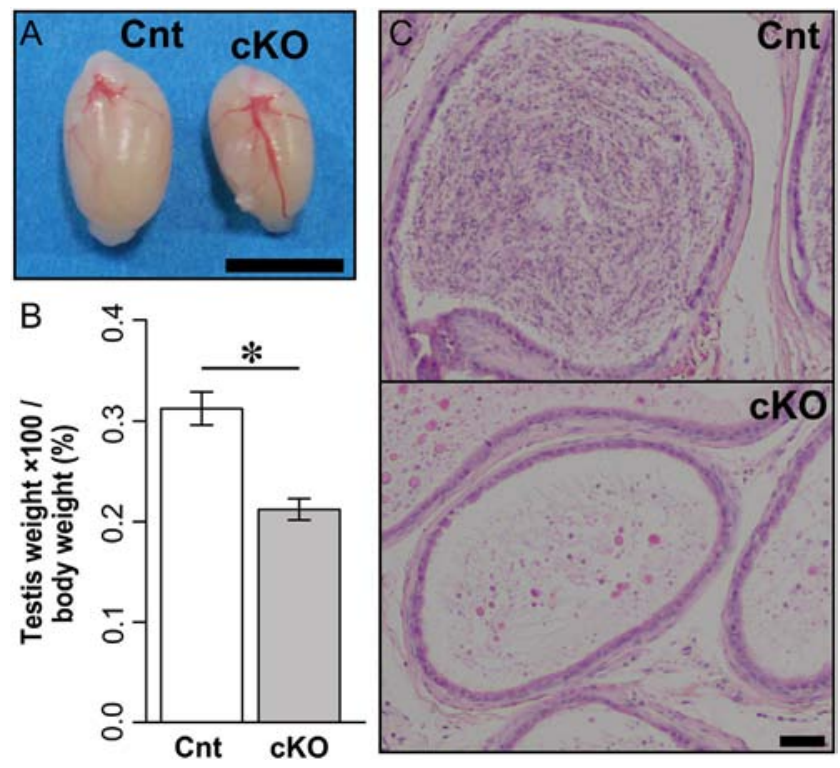

Figure 2 Decreased size of testes and low sperm production in 12-week-old Usp9x conditional knockout mice. (A) Gross appearance of Cnt and Usp9x cKO mouse testes. (B) The value of testis weight $\times 100 /$ body weight (\%) of Cnt and Usp9x cKO mice. $* P<0.05$ ( $n=3$ mice) (C) PAS-stained paraffin sections of $\mathrm{Cnt}$ and Usp9x cKO mouse epididymides. Scale bars, $5 \mathrm{~mm}$ in A; $50 \mu \mathrm{m}$ in C.

and Usp9x $x^{\text {loxp/y }}$ (Cnt) male founders were produced by the crossing between Vasa-Cre males and Usp9x $x^{\text {loxP/loxP }}$ females, and then, their reproductive phenotypes were examined. The testes of 12-week-old Usp9x cKO mice were significantly decreased in weight, as compared to those of the Cnt males (Fig. 2A and B). Few spermatozoa were observed in the caudal epididymides of the adult Usp9x cKO (Fig. 2C). As for their fertility, the Usp9x cKO males were able to properly make the vaginal plugs in wild-type females, but these females did not have any signs of pregnancy and produced no offspring ( $n=6$ males). This is in contrast to the $2.2 \pm 0.3$ pregnant females with a normal number of offspring produced following mating to the Cnt males. These data indicate that Usp9x cKO males are infertile presumably due to few epididymal spermatozoa.

\section{Defective spermatogenesis with reduced numbers of spermatocytes and subsequent failure of spermiation in Usp9x conditional knockout testes}

Histopathological examination revealed aberrant spermatogenesis with reduced number of spermatogenic cells in 12-week-old Usp9x cKO mouse testes (Fig. 3A). Such histopathological phenotypes were similar to those observed in 24-week-old and 1-year-old Usp9x cKO testes (Fig. 3B and C), suggesting certain defects in meiotic/post-meiotic germ cells rather than the defective maintenance of spermatogonial stem/progenitor cells in the Usp9x cKO testes. By the morphometric analyses using the transverse tubular sections of the 12-week-old testes, aberrant seminiferous tubules lacking some spermatogenic cells were $85.0 \pm 5.1 \%$ of transverse sections of seminiferous tubules. In these abnormal tubules, $3.3 \pm 1.7 \%$ of the tubules contained both round and elongated spermatids but completely lacked the spermatocytes; $68.8 \pm 7.7 \%$ of the tubules included a few spermatocytes and also a few spermatids; and $13.0 \pm 2.1 \%$ of the tubules showed the almost complete lack of the meiotic and post-meiotic spermatogenic cells in the adluminal compartment (Fig. 3D).

Anti-SCP3 immunostaining revealed that the relative number of spermatocytes in Usp9x cKO testes was significantly reduced to three-fourths of the control testes (SCP3-positive cell number per tubule: $70.7 \pm 3.4$ in cKO vs $93.0 \pm 3.9$ in $\mathrm{Cnt} ; n=3$ ), albeit of no appreciable differences in their signal intensities between the Usp9x cKO and control testes (Fig. 3E). Immunostaining of g-H2AX (the phosphorylated form of histone H2AX; localized at sites of meiotic recombination that occur during leptotene stage and later at $\mathrm{XY}$ body during pachytene stage (Mahadevaiah et al. 2001, FernandezCapetillo et al. 2003)) revealed that the spermatocytes, which passed through meiotic differentiation and remained in the affected seminiferous tubules showed no appreciable defects in recombination and formation of $\mathrm{XY}$ body (Fig. 3F). Immunostaining of Hsc70t (a specific marker for the cytoplasm of elongating spermatids (Tsunekawa et al. 1999)) showed proper luminal distribution and intensities of Hsc70t-positive signals in some seminiferous tubules of the Usp9x cKO testes (Fig. 3G). However, some affected spermatids showed aberrant condensed Hsc70t-positive signals as a residual body-like structure in the Usp9x cKO seminiferous tubules (arrowheads and inset ' $a$ ' in Fig. 3G), together with the mislocation of the fully elongated spermatids attached with Hsc70t-positive signals near the basal compartment (inset ' $b$ ' of Fig. 3G). Histopathological analysis using PAS-stained sections confirmed that some fully completed spermatozoa were abnormally retained in the seminiferous tubules containing the elongating spermatids at step 9-10 (Fig. 3H), suggesting some defects in the processes of spermiogenesis and/ or spermiation of the Usp9x cKO testes. Moreover, some elongated spermatids were degenerating and occasionally misaligned near the basement membrane (circles in right panel of Fig. $3 \mathrm{H}$ ), suggesting Sertoli cell phagocytosis of aberrant spermatids.

\section{Increased apoptotic cell death in the early spermatocytes of Usp9x conditional knockout testes}

Next, in order to examine the initial defects of Usp9x-null germ cells, we spatiotemporally observed HE-stained paraffin sections. No appreciable defects were observed until 1-week-old stage (data not shown), but in 2-week-old stage, coinciding with the appearance of early spermatocytes in postnatal 

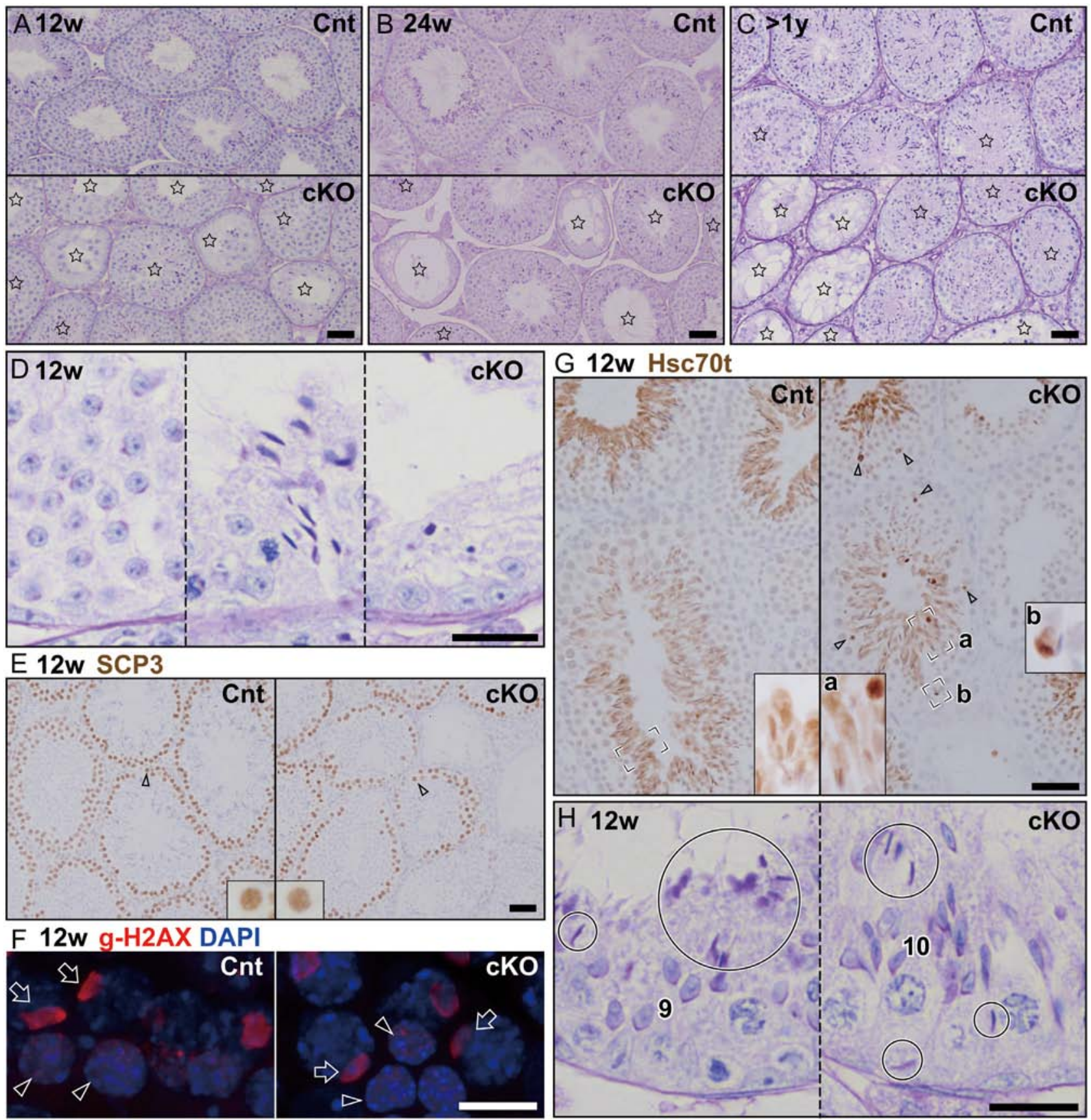

Figure 3 Defective spermatogenesis in Usp9x conditional knockout mice. (A, B, C and D) PAS-stained paraffin sections of 12-week-old (A, D), 24-week-old (B) and 1-year-old (C) Cnt and Usp9x cKO mouse testes. Stars in A, B and C indicate aberrant seminiferous tubules lacking some spermatogenic cells. (E) Anti-SCP3 (brown)-stained paraffin sections of 12-week-old Cnt and Usp9x cKO testes. Insets are the higher magnified images of the cells indicated by arrowheads. (F) Anti-g-H2AX (red)-stained cryosections of 12-week-old Cnt and Usp9x cKO testes. DNA was stained by DAPI. Arrows show XY body of pachytene spermatocytes and arrowheads show leptotene sprematocytes. (G, H) Anti-Hsc70t (brown; $\mathrm{G})$ and PAS (H) stained paraffin sections of 12-week-old Cnt and Usp9x cKO testes. Each arrowhead shows an aberrant condensed residual body-like structure that is positive for Hsc70t staining. Insets are the higher magnified images indicated by broken rectangles. Step 9 and step 10 spermatids are shown with Arabic numerals, and the mislocation of fully elongated spermatids and spermatozoa is indicated by circles in $\mathrm{H}$. Scale bars, $50 \mu \mathrm{m}$ in $\mathrm{A}, \mathrm{B}, \mathrm{C}, \mathrm{E}, \mathrm{G} ; 20 \mu \mathrm{m}$ in $\mathrm{D}, \mathrm{H} ; 10 \mu \mathrm{m}$ in $\mathrm{F}$.

development, apoptotic cells were frequently detected in Usp9x cKO mouse seminiferous tubules (Fig. 4A). Moreover, TUNEL analysis showed no differences of TUNEL-positive cell number between the Usp9x cKO and Cnt mice in the newborn and 1-week-old stages, but the Usp9x cKO testes showed a significant 

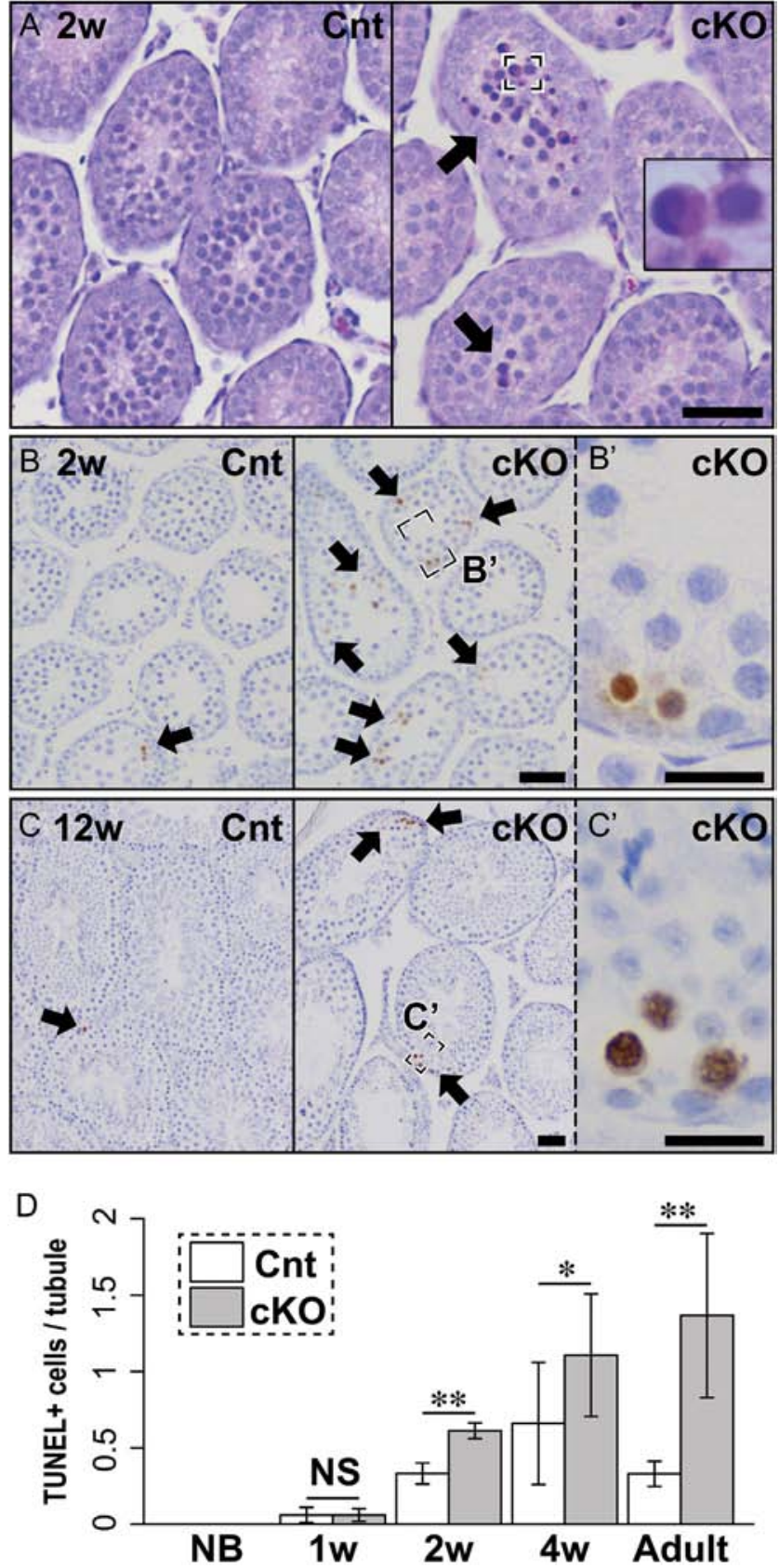

Figure 4 Spatiotemporal patterns of apoptotic cell death in Usp9x conditional knockout testes. (A) HE-stained paraffin sections of 2-week-old Cnt and Usp9x cKO mouse testes. Arrows show apoptotic cells. Inset is the higher magnified image indicated by broken rectangle. (B, C) TUNEL-stained paraffin sections of 2-weekold (B) and 12-week-old (C) mouse testes. TUNEL-positive cells (arrows) were found in the sections. $\mathrm{B}^{\prime}$ and $\mathrm{C}^{\prime}$ are the higher magnified images indicated by broken rectangles in Usp9x cKO of $\mathrm{B}$ and $C$ respectively. (D) The numbers of TUNEL-positive cells per tubule ( $n=2$ (newborn), $n=3$ (1-week-old, 4-week-old), $n=5$ (2-week-old), $n=6$ (adult)). NS, not significant; ${ }^{*} P<0.05 ;{ }^{* *} P<0.01$. Scale bars, $50 \mu \mathrm{m}$ in $\mathrm{A}, \mathrm{B}$ and $\mathrm{C} ; 20 \mu \mathrm{m}$ in $\mathrm{B}^{\prime}, \mathrm{C}^{\prime}$. increase of TUNEL-positive spermatogenic cells at 2-week-old and later stages (Fig. 4B, C and D). At 2-week-old stage, clusters of several TUNEL-positive spermatogenic cells were frequently observed in the luminal region nearest to the basal compartment (i.e., Sertoli cell nuclei) in the Usp9x cKO testes throughout the stages from 2- to 12-week-old (arrows in Fig. 4B and $\mathrm{C}$ ), suggesting apoptotic cell death around early spermatocyte stages. These data clearly agree with the present histological observation showing the frequent loss of the spermatocytes in the Usp9x cKO testes (Fig. 3A, B, C, D and E).

\section{Proper maintenance of the Usp9x-null spermatogonia in the basal compartment of the seminiferous tubules}

Next, we examined the phenotype of the Usp9xnull spermatogonia that are located in the basal compartment of the seminiferous tubules. Whole-mount staining of anti-GFRA1 antibody, a specific marker for the spermatogonial stem/progenitor cells (Hara et al. 2014), showed proper localization of GFRA1-positive spermatogonia in the basal compartment even in 1-yearold cKO testes (Fig. 5A), suggesting no appreciable defects in the spermatogonial stem/progenitor cells in Usp9x cKO testes. This is consistent with the partial spermatogenic patches containing spermatocytes and/or spermatids in some areas of Usp9x cKO testes even at 1 -year-old stage (Fig. 3C). The numbers of undifferentiated spermatogonia were quantitatively examined by using anti-PLZF staining, another marker for undifferentiated spermatogonia (Costoya et al. 2004). At 12-week-old, there was no significant difference in the number of PLZF-positive spermatogonia between Usp9x cKO testes and the Cnt testes of the littermates (PLZF-positive cell number per tubule: $9.2 \pm 1.2$ in cKO vs $10.8 \pm 1.0$ in Cnt; $n=3)$. Moreover, double staining of PLZF and Ki-67 (cell proliferation marker (Whitfield et al. 2006)) revealed no appreciable defects in Ki-67-positive cell ratio per PLZF-positive cells in cKO testes $(73.1 \pm 1.6 \%$ in cKO vs $75.5 \pm 2.8 \%$ in Cnt; $n=3$; Fig. $5 \mathrm{~B}$ ). Immunostaining using anti-KIT (a specific marker for differentiating spermatogonia (Zhang et al. 2011)) and anti-SOX9 (a marker for Sertoli cells (Kent et al. 1996)) antibodies showed no appreciable defects in the KIT-positive spermatogonia in the basal compartment of Usp9x cKO testes (KIT-positive cell number per SOX9-positive Sertoli cell: $0.84 \pm 0.08$ in cKO vs $0.97 \pm 0.12$ in Cnt; $n=3$; Fig. 5C). Taken together, these data suggest that Usp9x may not be essential for the maintenance and proliferation of the spermatogonia in mouse spermatogenesis, despite its predominant expression in them. 

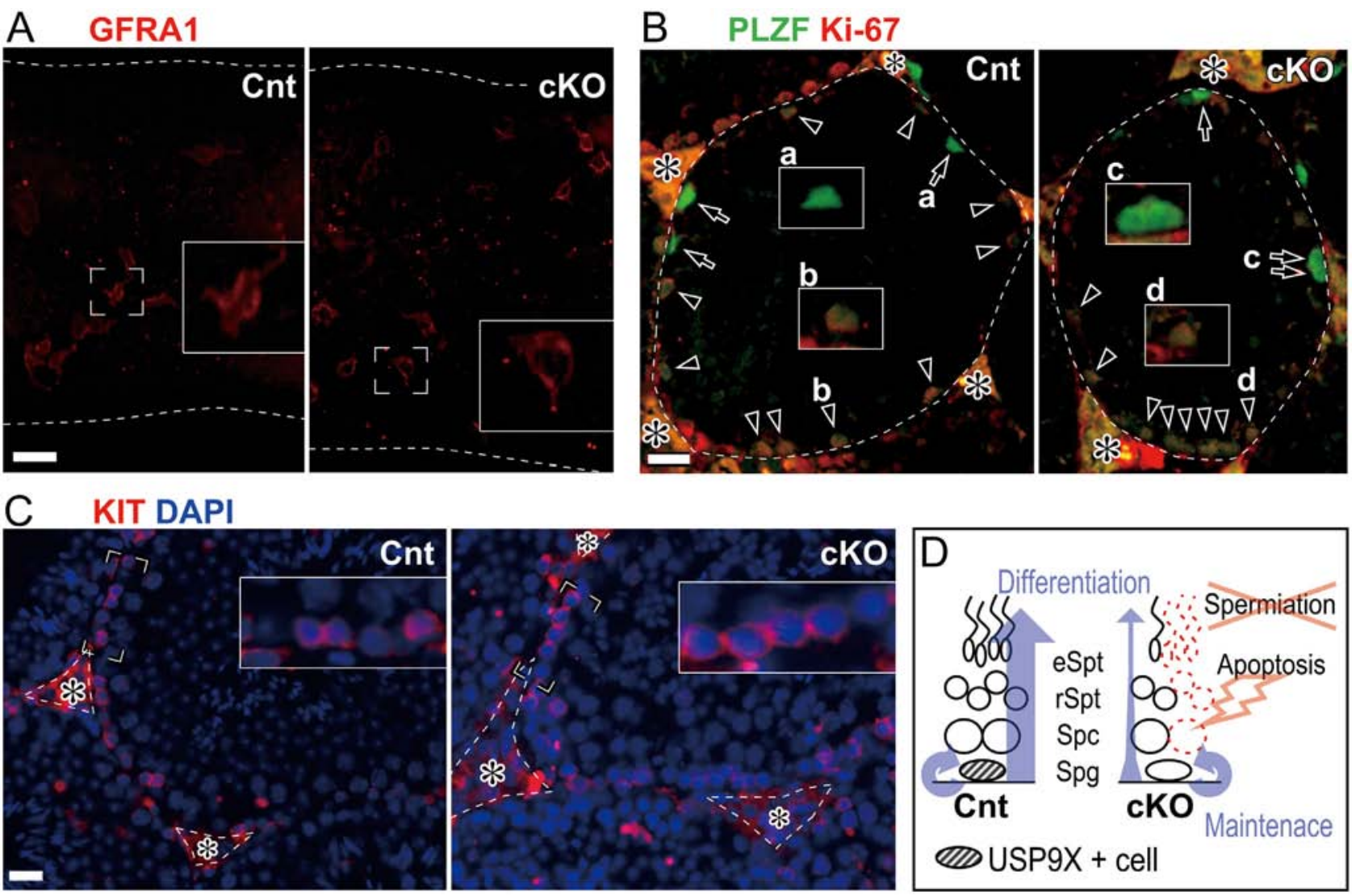

Figure 5 Proper maintenance of spermatogonia in Usp9x conditional knockout mice. (A) Anti-GFRA1 (red) whole-mount immunostaining of seminiferous tubules of 1-year-old Cnt and Usp9x cKO mice. Insets are the higher magnified images indicated by broken rectangles. (B) Anti-PLZF (green) and anti-Ki67 (red) double staining of paraffin sections of 12-week-old Cnt and Usp9x cKO mouse testis. PLZF single positive cells (arrows) and PLZF, Ki67 double positive cells (arrowheads) are observed in the seminiferous tubules. Insets are the higher magnified images indicated by the same alphabets. (C) Anti-KIT (red) and anti-SOX9 (not shown) double staining of cryosections of 12-week-old Cnt and Usp9x cKO mouse testis. DNA was stained by DAPI. Insets are the higher magnified images indicated by broken rectangles. (D) Schematic representation of the expression of USP9X and phenotypes of conditional knockout mice. Broken line, outline of seminiferous tubule in A and B, outline of interstitium in C; *, signals outside the seminiferous tubules; Spg, spermatogonium; Spc, spermatocyte; rSpt, round spermatid; eSpt, elongated spermatid. Scale bars, $20 \mu \mathrm{m}$.

\section{Discussion}

The present study detected considerable amount of USP9X-positive signals in the cytoplasm of gonocytes and spermatogonia during newborn to adult stages (Fig. 1). On the other hand, the meiotic and post-meiotic germ cells were USP9X negative, except for early spermatocytes in the 1st wave of spermatogenesis. Such a loss of USP9X expression soon after the meiotic entry may be caused by meiotic sex chromosome inactivation characterized by a specialized nuclear territory known as the $X Y$ body (Handel 2004), in which the Usp9x locus undergoes the transcriptional silencing at least from leptotene spermatocytes in mouse spermatogenesis (Mahadevaiah et al. 2008). Taken together, these data suggest the potential function of USP9X in the testicular germ cells mainly before the early phase of meiosis. In addition, no appreciable USP9X-positive signals were previously shown to be detected in the spermatogenic cells in postnatal and adult testes (Noma et al. 2002, Sato et al. 2004). This discrepancy may be due to the lower sensitivity of the previous antibody to detect the USP9X-positive signals.

A conditional deletion of Usp9x in testicular germ cells showed a partial loss of spermatocytes and subsequent defective spermiogenesis in Usp9x cKO testes (Figs 3 and 4). The first aberrant phenotypes at early spermatocyte stage were observed continuously from the immature, young adult to aged adult testes (from 2-week to 1-year-old). Moreover, TUNEL-positive apoptotic spermatogenic cells were observed in the adluminal area nearest to the basal compartment throughout the postnatal to adult stages. On the other hand, the maintenance of undifferentiated GFRA1positive/PLZF-positive and differentiating KIT-positive spermatogonia appeared to be normal, despite the dominant expression of USP9X in these spermatogonia. 
Therefore, these data indicate that a loss of function of spermatogonial USP9X leads to the defective transition from the mitotic to meiotic phases and/or defective maintenance of early meiotic phase in Usp9x cKO testes. This further suggests that the potential targets of USP9X may be involved in these early meiotic phases by protecting their degradation in the spermatogonia and early spermatocytes.

Recently, it becomes clear that USP9X is involved in various normal developmental processes and some abnormal disease conditions via stabilizing their target molecules (Murtaza et al. 2015). The previous studies showed that USP9X is able to stabilize ASK-1/DLK and protect them from proteasomal degradation in the pro-apoptotic JNK signaling cascade (Nagai et al. 2009, Huntwork-Rodriguez et al. 2013), in addition to the activation of anti-apoptotic factor, MCL1 and survivin (Vong et al. 2005, Schwickart et al. 2010). Moreover, $\beta$-catenin is one of the famous targets of USP9X (Taya et al. 1999). $\beta$-catenin has been reported to have important roles in germ cell differentiation (Chang et al. 2011) and maintenance of spermatogonia via regulation of the canonical Wnt signaling pathway (Takase \& Nusse 2016). It is possible that the imbalance in these signaling pathways may contribute to the present phenotypes showing increased apoptotic cell death at early spermatocyte stages, together with subsequent aberrant spermiogenesis in the Usp9x cKO testes.

One of the potential targets of USP9X may be VASA (formally known as DDX4 or MVH in mice), since Vasa is demonstrated as a target of Drosophila Faf, the functional orthologue of USP9X (Liu et al. 2003). Moreover, VASA proteins are well known to be a germ cell-specific component with high sequence conservation from Drosophila to mammals (Fujiwara et al. 1994). Mouse VASA is specifically expressed in germ cell lineage at stages from the PGCs in embryonic gonads and from spermatogonia to round spermatids during postnatal spermatogenesis (Toyooka et al. 2000). Mouse VASA is considered to be important in silencing retrotransposons and protecting germ cells (Kuramochi-Miyagawa et al. 2010). In fact, a deletion of mouse VASA leads to severe defects in spermatogenesis, in which almost all of premeiotic germ cells cease their differentiation at early spermatocyte stage and undergo apoptotic cell death (Tanaka et al. 2000). These meiotic defects in Vasanull germ cells are reminiscent of the apoptotic cell death in early spermatocytes of the Usp9x cKO testes, which further suggests the potential roles of USP9X in the spermatogenesis through the VASA stabilization. To elucidate the mechanisms of the exact actions of USP9X in these cellular events, the identification of the exact target molecules of USP9X including VASA must be required in future studies.

\section{Supplementary data}

This is linked to the online version of the paper at http://dx.doi. org/10.1530/REP-17-0184.

\section{Declaration of interest}

The authors declare that there is no conflict of interest that could be perceived as prejudicing the impartiality of the research reported.

\section{Funding}

This work was mainly supported by Grants-in-Aid for Scientific Research (KAKENHI) (\#24228005, \#26114504, \#16H01251). S A Wood acknowledges support from NHMRC project grant 1009248. K Kishi is DC1 JSPS Research Fellows.

\section{Acknowledgments}

The authors wish to thank Drs Hiroyuki Sumitomo, Hiroki Higashiyama, Yoshiko Kuroda, Yoshikazu Hirate, Kento Miura, Koji Hayakawa for their helpful supports and advice, and Itsuko Yagihashi and Yuki Uchiyama for their secretarial and technical supports.

\section{References}

Aiyama Y, Tsunekawa N, Kishi K, Kawasumi M, Suzuki H, Kanai-Azuma M, Kurohmaru M \& Kanai Y 2015 A niche for GFRalpha1-positive spermatogonia in the terminal segments of the seminiferous tubules in hamster testes. Stem Cells 33 2811-2824. (doi:10.1002/stem.2065)

Bose R, Manku G, Culty M \& Wing SS 2014 Ubiquitin-proteasome system in spermatogenesis. Advances in Experimental Medicine and Biology 759 181-213. (doi:10.1007/978-1-4939-0817-2_9)

Chang YF, Lee-Chang JS, Harris KY, Sinha-Hikim AP \& Rao MK 2011 Role of beta-catenin in post-meiotic male germ cell differentiation. PLOS ONE 6 e28039. (doi:10.1371/journal.pone.0028039)

Chen X, Overstreet E, Wood SA \& Fischer JA 2000 On the conservation of function of the Drosophila fat facets deubiquitinating enzyme and Fam, its mouse homolog. Development Genes and Evolution 210 603-610. (doi:10.1007/s004270000109)

Costoya JA, Hobbs RM, Barna M, Cattoretti G, Manova K, Sukhwani M, Orwig KE, Wolgemuth DJ \& Pandolfi PP 2004 Essential role of Plzf in maintenance of spermatogonial stem cells. Nature Genetics $\mathbf{3 6}$ 653-659. (doi:10.1038/ng1367)

Fernandez-Capetillo O, Mahadevaiah SK, Celeste A, Romanienko PJ, Camerini-Otero RD, Bonner WM, Manova K, Burgoyne P \& Nussenzweig A $2003 \mathrm{H} 2 \mathrm{AX}$ is required for chromatin remodeling and inactivation of sex chromosomes in male mouse meiosis. Developmental Cell 4 497-508. (doi:10.1016/S1534-5807(03)00093-5)

Fischer-Vize JA, Rubin GM \& Lehmann R 1992 The fat facets gene is required for Drosophila eye and embryo development. Development 116 985-1000.

Fujiwara $Y$, Komiya T, Kawabata H, Sato M, Fujimoto H, Furusawa M \& Noce T 1994 Isolation of a DEAD-family protein gene that encodes a murine homolog of Drosophila vasa and its specific expression in germ cell lineage. PNAS 91 12258-12262. (doi:10.1073/pnas.91.25.12258)

Gallardo T, Shirley L, John GB \& Castrillon DH 2007 Generation of a germ cell-specific mouse transgenic Cre line, Vasa-Cre. Genesis 45 413-417. (doi:10.1002/dvg.20310) 
Handel MA 2004 The XY body: a specialized meiotic chromatin domain. Experimental Cell Research 296 57-63. (doi:10.1016/j. yexcr.2004.03.008)

Hara K, Nakagawa T, Enomoto H, Suzuki M, Yamamoto M, Simons BD \& Yoshida S 2014 Mouse spermatogenic stem cells continually interconvert between equipotent singly isolated and syncytial states. Cell Stem Cell 14 658-672. (doi:10.1016/j.stem.2014.01.019)

Hou CC \& Yang WX 2013 New insights to the ubiquitin-proteasome pathway (UPP) mechanism during spermatogenesis. Molecular Biology Reports 40 3213-3230. (doi:10.1007/s11033-012-2397-y)

Huntwork-Rodriguez S, Wang B, Watkins T, Ghosh AS, Pozniak CD, Bustos D, Newton K, Kirkpatrick DS \& Lewcock JW 2013 JNK-mediated phosphorylation of DLK suppresses its ubiquitination to promote neuronal apoptosis. Journal of Cell Biology 202 747-763. (doi:10.1083/ jcb.201303066)

Kanai-Azuma M, Mattick JS, Kaibuchi K \& Wood SA 2000 Co-localization of FAM and AF-6, the mammalian homologues of Drosophila faf and canoe, in mouse eye development. Mechanisms of Development 91 383-386. (doi:10.1016/S0925-4773(99)00294-4)

Kent J, Wheatley SC, Andrews JE, Sinclair AH \& Koopman P 1996 A malespecific role for SOX9 in vertebrate sex determination. Development 122 2813-2822.

Kuramochi-Miyagawa S, Watanabe T, Gotoh K, Takamatsu K, Chuma S, Kojima-Kita K, Shiromoto Y, Asada N, Toyoda A, Fujiyama A et al. 2010 $\mathrm{MVH}$ in piRNA processing and gene silencing of retrotransposons. Genes and Development 24 887-892. (doi:10.1101/gad.1902110)

Lasko P 2013 The DEAD-box helicase Vasa: evidence for a multiplicity of functions in RNA processes and developmental biology. Biochimica et Biophysica Acta 1829 810-816. (doi:10.1016/j.bbagrm.2013.04.005)

Liu N, Dansereau DA \& Lasko P 2003 Fat facets interacts with vasa in the Drosophila pole plasm and protects it from degradation. Current Biology 13 1905-1909. (doi:10.1016/j.cub.2003.10.026)

Luddi A, Margollicci M, Gambera L, Serafini F, Cioni M, De Leo V, Balestri P \& Piomboni P 2009 Spermatogenesis in a man with complete deletion of USP9Y. New England Journal of Medicine 360 881-885. (doi:10.1056/ NEJMoa0806218)

Mahadevaiah SK, Turner JM, Baudat F, Rogakou EP, de Boer P, BlancoRodriguez J, Jasin M, Keeney S, Bonner WM \& Burgoyne PS 2001 Recombinational DNA double-strand breaks in mice precede synapsis. Nature Genetics 27 271-276. (doi:10.1038/85830)

Mahadevaiah SK, Bourc'his D, de Rooij DG, Bestor TH, Turner JM \& Burgoyne PS 2008 Extensive meiotic asynapsis in mice antagonises meiotic silencing of unsynapsed chromatin and consequently disrupts meiotic sex chromosome inactivation. Journal of Cell Biology 182 263-276. (doi:10.1083/jcb.200710195)

Murtaza M, Jolly LA, Gecz J \& Wood SA 2015 La FAM fatale: USP9X in development and disease. Cellular and Molecular Life Sciences 72 2075-2089. (doi:10.1007/s00018-015-1851-0)

Nagai H, Noguchi T, Homma K, Katagiri K, Takeda K, Matsuzawa A \& Ichijo H 2009 Ubiquitin-like sequence in ASK1 plays critical roles in the recognition and stabilization by USP9X and oxidative stress-induced cell death. Molecular Cell 36 805-818. (doi:10.1016/j.molcel.2009.10.016)

Noma T, Kanai Y, Kanai-Azuma M, Ishii M, Fujisawa M, Kurohmaru M, Kawakami H, Wood SA \& Hayashi Y 2002 Stage- and sex-dependent expressions of Usp9x, an X-linked mouse ortholog of Drosophila Fat facets, during gonadal development and oogenesis in mice. Mechanisms of Development 119 (Supplement 1) S91-S95. (doi:10.1016/S09254773(03)00098-4)

Russell L, Ettlin R, Sinha Hikim A \& Clegg E 1990 Histological and Histopathological Evaluation of the Testis. Clearwater: Cache River Press.

Ryu KY, Sinnar SA, Reinholdt LG, Vaccari S, Hall S, Garcia MA, Zaitseva TS, Bouley DM, Boekelheide K, Handel MA et al. 2008 The mouse polyubiquitin gene Ubb is essential for meiotic progression. Molecular and Cellular Biology 28 1136-1146. (doi:10.1128/MCB.01566-07)
Sato T, Kanai Y, Noma T, Kanai-Azuma M, Taya S, Matsui T, Ishii M, Kawakami H, Kurohmaru M, Kaibuchi K et al. 2004 A close correlation in the expression patterns of Af- 6 and Usp9x in Sertoli and granulosa cells of mouse testis and ovary. Reproduction 128 583-594. (doi:10.1530/ rep.1.00060)

Schwickart M, Huang X, Lill JR, Liu J, Ferrando R, French DM, Maecker H, O'Rourke K, Bazan F, Eastham-Anderson J et al. 2010 Deubiquitinase USP9X stabilizes MCL1 and promotes tumour cell survival. Nature $\mathbf{4 6 3}$ 103-107. (doi:10.1038/nature08646)

Stegeman S, Jolly LA, Premarathne S, Gecz J, Richards LJ, MackaySim A \& Wood SA 2013 Loss of Usp9x disrupts cortical architecture, hippocampal development and TGFbeta-mediated axonogenesis. PLoS ONE 8 e68287. (doi:10.1371/journal.pone.0068287)

Suresh B, Lee J, Hong SH, Kim KS \& Ramakrishna S 2015 The role of deubiquitinating enzymes in spermatogenesis. Cellular and Molecular Life Sciences 72 4711-4720. (doi:10.1007/s00018-015-2030-z)

Takase HM \& Nusse R 2016 Paracrine Wnt/beta-catenin signaling mediates proliferation of undifferentiated spermatogonia in the adult mouse testis. PNAS 113 E1489-E1497. (doi:10.1073/pnas.1601461113)

Tanaka SS, Toyooka Y, Akasu R, Katoh-Fukui Y, Nakahara Y, Suzuki R, Yokoyama M \& Noce T 2000 The mouse homolog of Drosophila Vasa is required for the development of male germ cells. Genes and Development 14 841-853.

Taya S, Yamamoto T, Kanai-Azuma M, Wood SA \& Kaibuchi K 1999 The deubiquitinating enzyme Fam interacts with and stabilizes beta-catenin. Genes to Cells 4 757-767. (doi:10.1046/j.1365-2443.1999.00297.x)

Toyooka Y, Tsunekawa N, Takahashi Y, Matsui Y, Satoh M \& Noce T 2000 Expression and intracellular localization of mouse Vasa-homologue protein during germ cell development. Mechanisms of Development 93 139-149. (doi:10.1016/S0925-4773(00)00283-5)

Tsunekawa N, Matsumoto M, Tone S, Nishida T \& Fujimoto H 1999 The Hsp70 homolog gene, Hsc70t, is expressed under translational control during mouse spermiogenesis. Molecular Reproduction and Development 52 383-391. (doi:10.1002/(SICl)1098-2795(199904)52:4<383::AIDMRD7>3.0.CO;2-Z)

Vong QP, Cao K, Li HY, Iglesias PA \& Zheng Y 2005 Chromosome alignment and segregation regulated by ubiquitination of survivin. Science $\mathbf{3 1 0}$ 1499-1504. (doi:10.1126/science.1120160)

Whitfield ML, George LK, Grant GD \& Perou CM 2006 Common markers of proliferation. Nature Reviews Cancer 6 99-106. (doi:10.1038/ nrc1802)

Yamauchi Y, Riel JM, Stoytcheva Z \& Ward MA 2014 Two Y genes can replace the entire $Y$ chromosome for assisted reproduction in the mouse. Science 343 69-72. (doi:10.1126/science.1242544)

Yoshida S, Sukeno M, Nakagawa T, Ohbo K, Nagamatsu G, Suda T \& Nabeshima Y 2006 The first round of mouse spermatogenesis is a distinctive program that lacks the self-renewing spermatogonia stage. Development 133 1495-1505. (doi:10.1242/dev.02316)

Yuan L, Liu JG, Zhao J, Brundell E, Daneholt B \& Hoog C 2000 The murine SCP3 gene is required for synaptonemal complex assembly, chromosome synapsis, and male fertility. Molecular Cell 5 73-83. (doi:10.1016/S1097-2765(00)80404-9)

Zhang L, Tang J, Haines CJ, Feng HL, Lai L, Teng X \& Han Y 2011 C-kit and its related genes in spermatogonial differentiation. Spermatogenesis $\mathbf{1}$ 186-194. (doi:10.4161/spmg.1.3.17760)

Received 27 March 2017

First decision 2 May 2017

Revised manuscript received 23 May 2017

Accepted 26 May 2017 